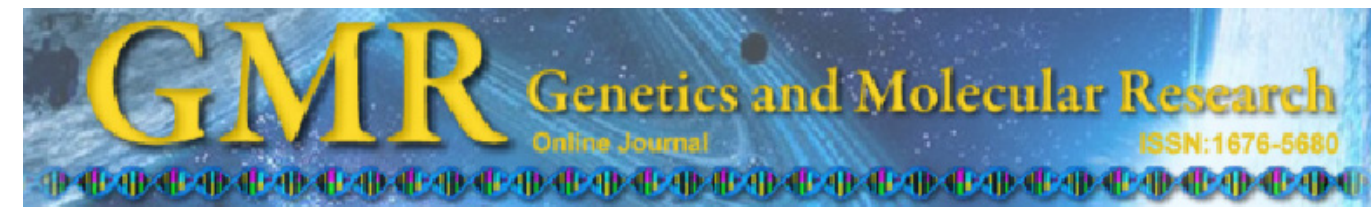

\title{
Absence of $\mathrm{SH} 2 \mathrm{~B} 3$ mutation in nonobese diabetic mice
}

\author{
Y.J. Li ${ }^{1,2 *}$, X.Y. Li ${ }^{1 *}$, X.R. Guo ${ }^{1}$, Y. Li ${ }^{1}$, B.F. Shen ${ }^{1}$, Y.C. Shi ${ }^{2}$ and \\ J.Y. Zhang ${ }^{1}$ \\ ${ }^{1}$ Department of Molecular Immunology, Institute of Basic Medical Sciences, \\ Beijing, P.R. China \\ ${ }^{2}$ Recearch Center of Molecular Biology, Inner Mongolia Medical College, \\ Hohhot, P.R. China \\ *These authors contributed equally to this study. \\ Corresponding authors: J.Y. Zhang / Y.C. Shi \\ E-mail: zhangjy@nic.bmi.ac.cn / ycshi5388@yahoo.com.cn
}

Genet. Mol. Res. 11 (2): 1266-1271 (2012)

Received September 19, 2011

Accepted January 30, 2012

Published May 9, 2012

DOI http://dx.doi.org/10.4238/2012.May.9.6

\begin{abstract}
Type 1 diabetes is a chronic progressive autoimmune disease characterized by mononuclear cell infiltration, with subsequent destruction of insulin-producing $\beta$-cells. Studies have identified strong associations between type 1 diabetes and several chromosome regions, including 12q24. Association between type 1 diabetes and $12 \mathrm{q} 24$ arises from SNP rs3184504; rs3184504 is a nonsynonymous SNP in exon 3 of $S H 2 B 3$ (also known as $L N K$ ). Nonobese diabetic (NOD) mice recapitulate many aspects of the pathogenesis of type 1 diabetes in humans and are therefore frequently used in studies addressing the cellular and molecular mechanisms of this disease. It is of interest to know whether there is a similar mutation of $S H 2 B 3$ in NOD mice. We found that the $S H 2 B 3$ mutation is absent in NOD mice. To our knowledge, this is the first report of the sequence and the protein levels of SH2B3 in NOD mice.
\end{abstract}

Key words: Mutation; SH2B3; Single nucleotide polymorphism; NOD 


\section{INTRODUCTION}

Type 1 diabetes (T1D) is a chronic progressive autoimmune disease characterized by mononuclear cell infiltration, dominated by pathogenic $\mathrm{T}$ cells, of the pancreatic islets, with subsequent destruction of insulin-producing $\beta$-cells (Hung et al., 2005). Although T1D is not a genetically predestined disease, an increased susceptibility to the disease can be inherited (Todd et al., 2007; Hunt et al., 2008). Genome-wide association studies have identified strong associations between T1D and several chromosome regions, including 12q24 (Todd et al., 2007; Hunt et al., 2008). An association between T1D and 12q24 arises from SNP rs3184504. rs3184504 is a nonsynonymous SNP in exon 3 of $S H 2 B 3$ (also known as $L N K$ ), leading to an $\mathrm{R} 262 \mathrm{~W}$ amino acid change in the pleckstrin homology domain. This domain may be important in plasma membrane targeting (Todd et al., 2007; Hunt et al., 2008). SH2B3 is strongly expressed in monocytes and dendritic cells, and to a lesser extent in resting B, T and natural killer cells (Li et al., 2000; Velazquez et al., 2002). SH2B3 regulates T-cell receptor, growth factor and cytokine receptor-mediated signaling, implicated in leukocyte and myeloid cell homeostasis (Li et al., 2000; Velazquez et al., 2002). Sh $2 b 3^{-/-}$mice have increased responses to multiple cytokines (Velazquez et al., 2002). However, it remains unknown how the R262W mutation of $S H 2 B 3$ contributes to the pathogenesis of T1D.

Nonobese diabetic (NOD) mice recapitulate many aspects of the pathogenesis of T1D in humans and are therefore frequently used in studies addressing the cellular and molecular mechanisms of T1D (Delovitch and Singh, 1997; Hung et al., 2005). It is of interest whether there is a similar mutation of $S H 2 B 3$ in NOD mice. If it is the case, NOD mice could be used as a good model to study how $S H 2 B 3$ mutation contributes to the pathogenesis of T1D.

\section{MATERIAL AND METHODS}

Female C57BL/6 mice and female NOD/LtJ mice were purchased from the Institutes of Experimental Animals, Academy of Chinese Medical Sciences (Beijing, China). All mice were maintained under specific pathogen-free conditions. All experiments were performed in accordance with institutional guidelines for animal care. Unless otherwise specified, mice were used between 3 and 4 weeks of age and were therefore nondiabetic. T1D develops in over $80 \%$ of NOD/LtJ female mice between 12-16 weeks of age in our colony.

Bone marrow-derived macrophages and dendritic cells (BMM and BMDC, respectively) were obtained by culturing the non-adherent bone marrow cells in RPMI 1640 medium containing 15\% (v/v) FBS, $2 \mathrm{mM}$ L-glutamine, $100 \mathrm{U} / \mathrm{mL}$ penicillin, $100 \mu \mathrm{g} / \mathrm{mL}$ streptomycin, and $50 \mu \mathrm{M} 2$-mercaptoethanol with $100 \mathrm{ng} / \mathrm{mL}$ M-CSF or $10 \mathrm{ng} / \mathrm{mL}$ GM-CSF and $10 \mathrm{ng} / \mathrm{mL}$ IL-4 for 7 days, as previously described (Zhang et al., 2010). FBS was from HyClone Laboratories (Logan, UT, USA). M-CSF was from Cetus (Emeryville, CA, USA). GM-CSF was from PeproTech (Rocky Hill, NJ, USA). IL-4 was from R\&D Systems (Minneapolis, MN, USA).

Thymocytes were obtained from single-cell suspensions of thymuses by direct depletion of red cells. CD4+ T cells were isolated (negative selection) from single-cell suspensions of spleens by using a CD4+ T cell isolation kit II and MS columns according to the manufacturer protocol. CD4+ T cell isolation kit II and MS columns were obtained from Miltenyi Biotech (Bergisch Gladbach, Germany).

RNA was extracted by using TRIzol reagent. cDNA was derived from $1 \mu \mathrm{g}$ total RNA 
by reverse transcription using M-MLV reverse transcriptase and oligo(dT) primer. TRIzol reagent, M-MLV reverse transcriptase, and oligo(dT) primer were from Invitrogen (Carlsbad, CA, USA). The following primer pairs were designed and used: $S H 2 B 3(1-517 \mathrm{bp}), 5^{\prime}$-ATGAA CGAGCCCACCGTGC-3' and 3'-CCTCCTTGAGCGCCTCAG-5'; SH2B3 (491-1168 bp), 5'-AGCCGCCCACTGAGGCGCTCAAGGAGGTCGTATTGC-3' and 3'-GCACCAGGCGT AAGTGCTTGGCTCTGC-5'; SH2B3 (1144-1647 bp), 5'-AGAGCCAAGCACTTACGC-3' and 3'-TCACACGTCTGCCTCTCTG-5'. All PCRs were carried out by a standard protocol for 30 cycles with $25 \mathrm{ng}$ cDNA. Aliquots of PCR mixtures were separated on a $1 \%(\mathrm{w} / \mathrm{v})$ agarose gel and visualized with UV light after ethidium bromide staining. The purified PCR products were ligated into a pGEM-T easy plasmid vector and subjected to sequencing. The synthesis of primers and sequencing were finished by Invitrogen.

Immunoblotting analysis was done as previously described (Cui et al., 2009). Briefly, cell lysates were prepared in ice-cold lysis buffer $(0.5 \%$ NP-40, $20 \mathrm{mM}$ Tris-Cl, $\mathrm{pH}$ 7.6, 250 $\mathrm{mM} \mathrm{NaCl}, 3 \mathrm{mM}$ EDTA, $3 \mathrm{mM}$ EGTA, $1 \mathrm{mM}$ sodium orthovanadate, $1 \mathrm{mM}$ DTT, $10 \mathrm{mM}$ PNPP, $10 \mu \mathrm{g} / \mathrm{mL}$ aprotinin). Cell lysates were then resolved by SDS-PAGE before transferring to nitrocellulose membranes. Nitrocellulose membranes were then incubated with $5 \%(\mathrm{w} / \mathrm{v})$ nonfat dry milk in washing buffer $(20 \mathrm{mM}$ Tris-Cl, $\mathrm{pH} 7.6,150 \mathrm{mM} \mathrm{NaCl}$, and $0.1 \%$ Tween 20) for $1 \mathrm{~h}$ at $37^{\circ} \mathrm{C}$ to block nonspecific protein binding. Primary antibodies were diluted in washing buffer containing $5 \%(\mathrm{w} / \mathrm{v})$ nonfat dry milk and applied to the membranes overnight at $4^{\circ} \mathrm{C}$. After extensive washing, the membranes were incubated with peroxidase-conjugated antibodies for $1 \mathrm{~h}$ at room temperature and washed again. Immunoreactive bands were visualized with the ECL chemiluminescence kit. Antibodies against $S H 2 B 3$ and actin were from Santa Cruz Biotechnology (Santa Cruz, CA, USA).

For flow cytometry analysis, the cells were washed with flow cytometry buffer $(2 \%$ FBS, $0.1 \% \mathrm{NaN}_{3}$ in PBS) twice, and then incubated with FITC- or PE-conjugated antibodies at $4^{\circ} \mathrm{C}$. Isotype antibodies were included as negative control. After washing with flow cytometry buffer, the cells were fixed with $1 \%(\mathrm{w} / \mathrm{v})$ paraformaldehyde in PBS and stored at $4{ }^{\circ} \mathrm{C}$. Flow cytometry was carried out on a Becton Dickinson FACSCalibur (BD Biosciences, Franklin Lakes, NJ, USA). FITC- or PE-conjugated antibodies were from eBioscience (San Diego, CA, USA).

\section{RESULTS AND DISCUSSION}

The $\mathrm{R} 262 \mathrm{~W}$ mutation in the human SH2B3 protein has been revealed to be implicated in T1D (Todd et al., 2007; Hunt et al., 2008). To analyze whether there is a similar mutation of $\mathrm{SH} 2 \mathrm{~B} 3$ in NOD mice, we purified the total RNA from NOD thymocytes and designed primers according to the reported sequence of C57BL/6 SH2B3 mRNA in NCBI. RT-PCR was successful (Figure 1). The purified PCR products were then ligated into a pGEM-T easy plasmid vector and sequenced. The sequencing data showed that the NOD SH2B3 mRNA has an open reading frame of $1647 \mathrm{bp}$, the same as its $\mathrm{C} 57 \mathrm{BL} / 6$ counterpart. However, no mutation was found in the open reading frame of the $S H 2 B 3$ mRNA in NOD mice (Figure 1).

Even though there is no mutation in the open reading frame of the SH2B3 mRNA in NOD mice, it is still possible that there is an alteration in the expression of $\mathrm{SH} 2 \mathrm{~B} 3$ contributing to the onset of diabetes in NOD mice. Because extensive studies have demonstrated that impaired T-cell regulation induction in the thymus and in the periphery plays a pivotal role in 
disease initiation and progression (D'Alise et al., 2008), thymocytes from 3-week-old C57BL/ B6 and NOD mice were first chosen to test this hypothesis. Immunoblotting analysis revealed that the protein level of SH2B3 in NOD thymocytes was similar to that in their C57BL/6 counterparts (Figure 2A). Furthermore, CD4+ splenic T cells were purified (Figure 2B), and immunoblotting analysis revealed that the protein level of SH2B3 in purified NOD CD4+ splenic T cells was similar to that in their C57BL/6 counterparts (Figure 2C).

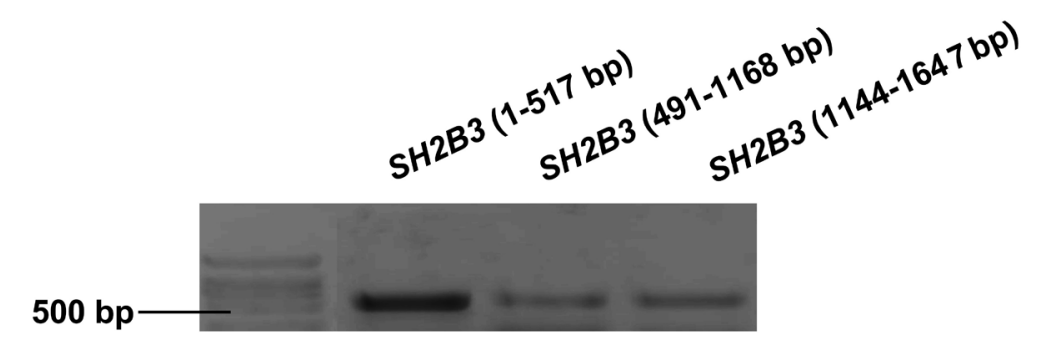

Figure 1. PCR amplification of $S H 2 B 3$ cDNA.

A

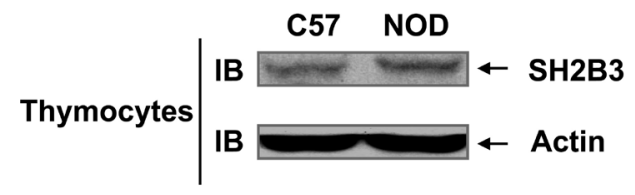

B

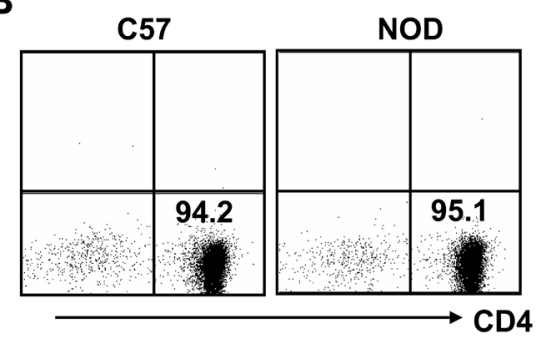

C

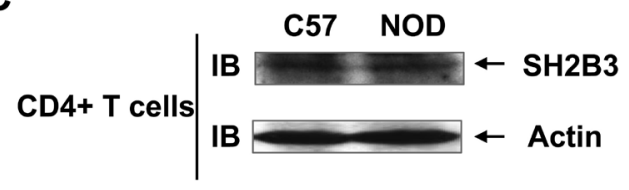

Figure 2. Similar protein levels of SH2B3 in C57BL/6 (C57) and nonobese diabetic (NOD) thymocytes and CD4+ $\mathrm{T}$ cells. A. and C. Comparison of the protein levels of SH2B3 in C57BL/6 and NOD thymocytes (A) and CD4+ $\mathrm{T}$ cells $(\mathbf{C})$ by immunoblotting (IB). B. Determination of the purity of CD4+ splenic T cells isolated. Data are representative of at least 6 individual experiments.

SH2B3 is strongly expressed in monocytes and dendritic cells ( $\mathrm{Li}$ et al., 2000; Velazquez et al., 2002). Several groups have demonstrated that compared to cells from reference strains of mice including C57BL/6, NOD macrophages and BMDC exhibit elevated IL12 production in response to various inflammatory stimuli such as LPS (Marleau et al., 2008). Thus, the expression of $\mathrm{SH} 2 \mathrm{~B} 3$ in NOD BMDC and BMM was explored. BMDC and BMM 
from C57BL/6 and NOD mice showed similar expression of the dentritic cell marker CD11c and macrophage marker F4/80, respectively (Figure 3A and C). Moreover, immunoblotting revealed that the protein levels of SH2B3 in NOD BMDC and BMM were similar to those in their $\mathrm{C} 57 \mathrm{BL} / 6$ counterparts (Figure 3B and D).

A

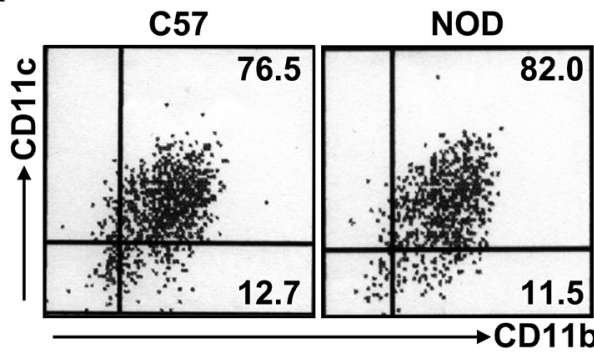

C

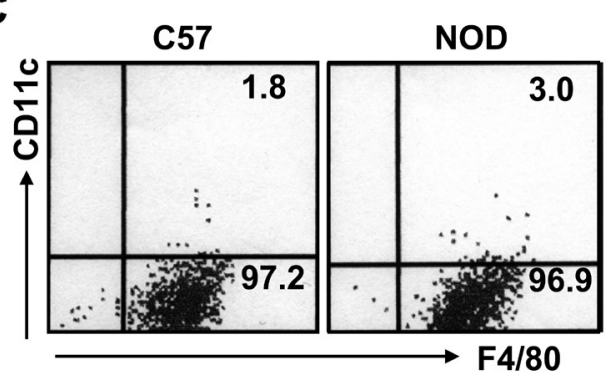

B

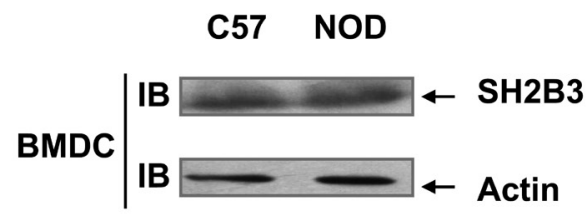

D

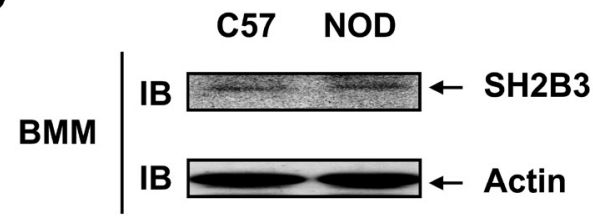

Figure 3. Similar protein levels of SH2B3 in C57BL/6 (C57) and nonobese diabetic (NOD) BMDC and BMM. A. and C. Determination of the protein levels of CD11c and F4/80 in C57BL/6 and NOD BMDC (A) and BMM (C), respectively. B. and D. Comparison of the protein levels of SH2B3 in C57BL/6 and NOD BMDC (B) and BMM (D) by immunoblotting (IB). BMM and BMDC = bone marrow-derived macrophages and dendritic cells, respectively. Data are representative of at least 6 individual experiments.

\section{CONCLUSIONS}

Collectively, our data suggest that NOD mice are not appropriate for use in the study of how the R262W mutation of $S H 2 B 3$ contributes to the pathogenesis of T1D. To our knowledge, we are the first to report that the sequence and expression of $\mathrm{SH} 2 \mathrm{~B} 3$ exhibit no significant alteration in NOD mice.

\section{ACKNOWLEDGMENTS}

Research supported by the Key Natural Science Program of Beijing (\#7101008) and the National Key Basic Research Program of China (\#2010CB911904).

\section{REFERENCES}

Cui J, Zhu N, Wang Q, Yu M, et al. (2009). p38 MAPK contributes to CD54 expression and the enhancement of phagocytic 
activity during macrophage development. Cell Immunol. 256: 6-11.

D'Alise AM, Auyeung V, Feuerer M, Nishio J, et al. (2008). The defect in T-cell regulation in NOD mice is an effect on the T-cell effectors. Proc. Natl. Acad. Sci. U. S. A. 105: 19857-19862.

Delovitch TL and Singh B (1997). The nonobese diabetic mouse as a model of autoimmune diabetes: immune dysregulation gets the NOD. Immunity 7: 727-738.

Hung JT, Liao JH, Lin YC, Chang HY, et al. (2005). Immunopathogenic role of TH1 cells in autoimmune diabetes: evidence from a T1 and T2 doubly transgenic non-obese diabetic mouse model. J. Autoimmun. 25: 181-192.

Hunt KA, Zhernakova A, Turner G, Heap GA, et al. (2008). Newly identified genetic risk variants for celiac disease related to the immune response. Nat. Genet. 40: 395-402.

Li Y, He X, Schembri-King J, Jakes S, et al. (2000). Cloning and characterization of human Lnk, an adaptor protein with pleckstrin homology and Src homology 2 domains that can inhibit T cell activation. J. Immunol. 164: 5199-5206.

Marleau AM, Summers KL and Singh B (2008). Differential contributions of APC subsets to T cell activation in nonobese diabetic mice. J. Immunol. 180: 5235-5249.

Todd JA, Walker NM, Cooper JD, Smyth DJ, et al. (2007). Robust associations of four new chromosome regions from genome-wide analyses of type 1 diabetes. Nat. Genet. 39: 857-864.

Velazquez L, Cheng AM, Fleming HE, Furlonger C, et al. (2002). Cytokine signaling and hematopoietic homeostasis are disrupted in Lnk-deficient mice. J. Exp. Med. 195: 1599-1611.

Zhang J, Zhu N, Wang Q, Wang J, et al. (2010). MEKK3 overexpression contributes to the hyperresponsiveness of IL-12overproducing cells and CD4+ T conventional cells in nonobese diabetic mice. J. Immunol. 185: 3554-3563. 